\section{Dificuldade em atividades de vida diária e necessidade de ajuda em idosos: discutindo modelos de distanciamento social com evidências da iniciativa ELSI-COVID-19}

\author{
Difficulty in performing activities of daily living \\ and the need for help in older adults: evidence \\ on social distancing models from the \\ ELSI-COVID-19 initiative
}

Dayane Capra de Oliveira 1 Cesar Messias de Oliveira 2 Maria Fernanda Lima-Costa 3,4

Tiago da Silva Alexandre 1,5,6

\section{Resumo}

Analisar se idosos com dificuldade ou necessidade de ajuda para desempenhar atividades básicas ou instrumentais de vida diária estão mais distanciados socialmente em tempos de COVID-19. Participaram das entrevistas telefônicas 4.035 idosos participantes da $2 \underline{a}$ onda do Estudo Longitudinal de Saúde dos Idosos Brasileiros (ELSI-Brasil). Classificou-se a dificuldade, necessidade e recebimento de ajuda em: (1) independentes; (2) com dificuldade e não precisa de ajuda; (3) com dificuldade, precisa e recebe ajuda de pessoa de dentro de casa; (4) com dificuldade, precisa e recebe ajuda de pessoa de fora de casa; e (5) com dificuldade, precisa, mas não recebe ajuda. O distanciamento social foi categorizado como não saiu de casa nos últimos 7 dias, saiu de casa para atividades essenciais e saiu de casa para atividades não essenciais. Modelo de regressão multinomial controlado por idade, sexo, escolaridade e região do Brasil foi utilizado. Permaneceram mais distanciados socialmente os idosos que apresentavam dificuldade, necessitavam e recebiam ajuda de pessoa de dentro (odds ratio - OR =2,34; IC95\%: 1,25-4,39) ou de fora de casa $(O R=3,94 ;$ IC95\%: 2,24-6,92). A idade aumentou a chance de não sair de casa $(O R=1,06$; IC95\%: 1,03-1,09) e ser homem a diminuiu $(O R=0,48$; IC95\%: 0,33-0,70). Viver na Região Sul aumentou a chance dos idosos saírem para atividades essenciais (OR =1,77; IC95\%: 1,01-3,10). Idosos com dificuldade, que precisam e recebiam ajuda de pessoas de dentro ou de fora de casa não saíram de casa nos últimos 7 dias. Mesmo com o distanciamento social, esses idosos podem não ter sua exposição ao COVID-19 reduzida, enfraquecendo a teoria do distanciamento social seletivo.

Isolamento Social; Incapacidade; COVID-19; Cuidado; Idoso

\author{
Correspondência \\ T. S. Alexandre \\ Departamento de Gerontologia, Universidade Federal de São \\ Carlos. \\ Rod. Washington Luís, Km 235, São Carlos, SP \\ 13565-905, Brasil. \\ tiagoalexandre@ufscar.br \\ 1 Programa de Pós-graduação em Fisioterapia, Universidade \\ Federal de São Carlos, São Carlos, Brasil. \\ 2 University College London, London, U.K. \\ 3 Instituto René Rachou, Fundação Oswaldo Cruz, Belo \\ Horizonte, Brasil. \\ 4 Programa de Pós-graduação em Saúde Púbica, Universidade \\ Federal de Minas Gerais, Belo Horizonte, Brasil. \\ 5 Programa de Pós-graduação em Gerontologia, Universidade \\ Federal de São Carlos, São Carlos, Brasil. \\ 6 Departamento de Gerontologia, Universidade Federal de São \\ Carlos, São Carlos, Brasil.
}




\section{Introdução}

Em 11 de março de 2020, a Organização Mundial da Saúde decretou a situação de pandemia causada por um novo coronavírus (SARS-CoV-2), agente etiológico da COVID-19 1,2,3. A COVID-19 é uma infecção do sistema respiratório que tem nas gotículas respiratórias um dos meios de transmissão 4 . Tal condição apresenta como sintomas: secreções nasais, tosse, dispneia, febre, cansaço para pequenos esforços e, ocasionalmente, diarreia e alterações no sistema nervoso central. Contudo, a COVID-19 pode também cursar sem a presença de sintomas 1,5,6.

Ainda que não exista uma vacina para a COVID-19 ou tratamento medicamentoso reconhecidamente comprovado 7 , diversas recomendações vêm sendo dadas à população para evitar a transmissão do vírus, sendo as principais: cobrir a boca e o nariz ao tossir/espirrar, uso de máscaras faciais, lavagem das mãos com frequência com água e sabão ou álcool em gel, bem como manter distanciamento social $8,9,10$.

Esse distanciamento consiste em diminuir a interação de indivíduos da comunidade com o intuito de desacelerar a transmissão do vírus e pode ocorrer de duas maneiras: o distanciamento social seletivo, uma estratégia recomendada especialmente para proteger as populações de maior risco como, por exemplo, idosos, gestantes e pessoas com comorbidades descompensadas como hipertensão arterial, diabetes e doenças cardiovasculares; e o distanciamento social ampliado, em que não há seleção de grupos prioritários 10,11,12. No Brasil, no início da pandemia, o distanciamento social ampliado vinha sendo praticado como uma estratégia de enfrentamento do vírus. Todavia, ao longo do tempo, ele vem sendo flexibilizado e alguns defendem que o distanciamento social seletivo fosse adotado em seu lugar.

Entretanto, há de se destacar que existe uma parcela importante da população idosa que apresenta dificuldades na execução de atividades instrumentais da vida diária (AIVD) 13, que indicam a capacidade do indivíduo em levar uma vida independente dentro da comunidade onde vive e/ou de atividades básicas da vida diária (ABVD) 14, que incluem tarefas relacionadas ao autocuidado e sobrevivência, e precisam de ajuda para realizá-las. Em geral, os que prestam ajudam a esses idosos são pessoas que residem no mesmo domicílio do idoso ou em outro domicílio, mas que vão até a casa desse idoso para prestar tal ajuda. Portanto, esse idosos com dificuldade em ABVD e/ou AIVD e que necessitam de ajuda podem estar expostos ao risco de serem contaminados pelas pessoas que lhes prestam ajuda numa situação de distanciamento social ampliado.

Dessa forma, para que pudéssemos ter informações para discutir a melhor forma de distanciamento social a ser considerada para a população idosa, principalmente a mais dependente, o objetivo do presente estudo foi analisar se os idosos com dificuldade para desempenhar ABVD e/ou AIVD e que necessitam de ajuda para realizar essas atividades estão mais distanciados socialmente neste período de pandemia de COVID-19.

\section{Método}

\section{Delineamento do estudo}

Trata-se de um estudo transversal, que utilizou dados do inquérito da 2a onda do Estudo Longitudinal da Saúde dos Idosos Brasileiros (ELSI-Brasil), conduzido em amostra nacional representativa da população brasileira com 50 anos ou mais. Para garantir a representatividade, o delineamento da amostra foi baseado em estágios de seleção, combinando municípios (unidade primária), setores censitários e domicílios. O ELSI-Brasil foi conduzido em 70 municípios, situados nas cinco grandes regiões do país 15. Uma descrição detalhada do estudo pode ser encontrada em outra publicação 16 e na homepage da pesquisa (http://elsi.cpqrr.fiocruz.br/). 


\section{Inquérito telefônico}

A 2a onda do ELSI-Brasil iniciou em agosto de 2019 e foi interrompida em 17 de março de 2020, em função da pandemia da COVID-19. Até a interrupção do campo, 9.177 pessoas haviam sido avaliadas na 2 a onda por meio de entrevistas e de medidas físicas, utilizando os mesmos instrumentos e procedimentos adotados para a coleta de dados do inquérito da linha de base. Todos os participantes da 2a onda do ELSI-Brasil foram elegíveis para o inquérito telefônico sobre a COVID-19 (iniciativa ELSICOVID-19). Entre os elegíveis, 6.149 (67\%) responderam à entrevista telefônica. As características dos participantes do inquérito telefônico foram semelhantes às da população brasileira com 50 anos ou mais em relação a todas as características sociodemográficas analisadas, tais como idade, sexo, número de moradores no domicilio, escolaridade, região de residência e tipo de residência (urbana/ rural). A entrevista telefônica foi realizada entre os dias 26 de maio e 8 de junho de 2020, incluindo informações sobre a adesão a medidas de prevenção (distanciamento social, uso de máscaras e higienização das mãos), razões para sair de casa quando este era o caso, dificuldades para a obtenção de medicamentos, diagnóstico médico para a COVID-19 e realização de testes confirmatórios, bem como uso de serviços de saúde. As chamadas telefônicas foram planejadas para serem curtas (duração de 5 minutos) e foram realizadas por entrevistadores previamente treinados, preferencialmente aqueles que realizaram as entrevistas domiciliares da 2 a onda do estudo.

Foram elegíveis para a presente análise todos os participantes do inquérito telefônico com 60 anos ou mais, compreendendo 4.292 indivíduos. Desses, 257 foram excluídos da amostra devido à falta de informações em pelo menos uma das covariáveis (idade, sexo, escolaridade e grande região) resultando numa amostra analítica final de 4.035 indivíduos.

As informações sobre idade, sexo, escolaridade, ABVD, AIVD, necessidade de ajuda e grande região brasileira onde foi realizada a entrevista foram obtidas da 2a onda do ELSI-Brasil. As informações acerca do distanciamento social foram obtidas por meio do inquérito telefônico ELSI-COVID-19 17.

O ELSI-Brasil e a iniciativa ELSI-COVID-19 foram aprovados pelo Comitê de Ética do Instituto René Rachou, Fundação Oswaldo Cruz (CAAE: 34649814.3.0000.509 e CAAE: 33492820.3.0000.5091, respectivamente).

\section{Atividades da vida diária}

As ABVD foram avaliadas pelo índice de Katz modificado 14 (banhar-se, alimentar-se, andar, transferir-se, vestir-se e usar o banheiro). As AIVD foram avaliadas por meio da escala de Lawton adaptada 18 (preparar refeições, usar transporte, fazer compras, usar o telefone, administrar o dinheiro e as medicações). Foi considerado se os indivíduos tinham dificuldades, se precisavam de ajuda e se recebiam ajuda para realizar as ABVD e AIVD. Dessa forma, os idosos foram classificados em cinco grupos: (1) independentes para ABVD e AIVD; (2) possuíam dificuldades para ABVD ou AIVD e não precisavam de ajuda; (3) possuíam dificuldades para ABVD ou AIVD, precisavam e recebiam ajuda de pessoa de dentro de casa; (4) possuíam dificuldades para ABVD ou AIVD, precisavam e recebiam ajuda de pessoa de fora de casa; e (5) possuíam dificuldades para ABVD ou AIVD, precisavam, mas não recebiam ajuda. A categoria dos indivíduos independentes foi considerada como referência.

\section{Estado de distanciamento social}

O estado de distanciamento social foi definido pela frequência e razões para ter saído de casa nos 7 dias precedentes. Foram consideradas razões não essenciais: fazer exercícios físicos, encontrar amigos ou outras razões não mencionadas; e as razões essenciais: comprar remédios, atendimento de saúde ou pagamento de contas. Sair de casa para trabalhar não foi considerada como atividade essencial neste estudo, uma vez que nosso objetivo é analisar a dificuldade em ABVD e/ou AIVD, bem como a necessidade de ajuda para desempenhá-las. Por fim, o estado de distanciamento social foi definido como: saiu para atividades não essenciais (grupo de referência); saiu para atividades essenciais e não saiu de casa nos últimos 7 dias. 


\section{Covariáveis}

As características sociodemográficas incluíram: idade (anos), sexo, escolaridade (analfabeto, 1-4 anos, 5-8 e > 8 anos) e grande região onde foi realizada a entrevista (Norte, Nordeste, Sudeste, Sul e Centro-oeste).

\section{Análises estatísticas}

Análises descritivas foram realizadas para a caracterização da amostra. As comparações entre os grupos em relação ao estado de distanciamento social foram feitas utilizando-se o teste de Wald e Rao-Scott (comparação de médias) e o teste do qui-quadrado com a correção Rao-Scott (comparação de proporções) e seus respectivos intervalos de 95\% de confiança (IC95\%). Para todas as análises foram considerados o efeito do delineamento da amostra e os pesos especificamente derivados para os respondentes à entrevista telefônica.

Um modelo de regressão multinomial controlado por idade, sexo, escolaridade e região do Brasil onde ocorreu a entrevista foi usado para verificar se os idosos com dificuldade para desempenhar atividades básicas (ABVD) ou instrumentais da vida diária (AIVD) e que necessitam de ajuda estavam mais distanciados socialmente neste período de pandemia de COVID-19. Para a interpretação dos resultados, aqueles que saíram para atividades não essenciais (desfecho) e aqueles sem dificuldade e necessidade de ajuda para ABVD e AIVD (exposição) foram considerados grupos de referência (odds ratio $-\mathrm{OR}=1,00)$. Um valor de $\mathrm{p}<0,05$ foi adotado para indicar significância estatística. Para o cálculo das probabilidades preditas da permanência em casa nos últimos 7 dias de acordo com a dificuldade, necessidade e recebimento de ajuda para ABVD ou AIVD, por idade, foi utilizado o modelo de regressão multinomial controlado por sexo, escolaridade e região do Brasil. O programa Stata $15 \mathrm{SE}$ (https://www.stata.com) foi usado em todas as análises.

\section{Resultados}

Dos 4.035 participantes, 37,2\% saíram para atividades essenciais e 48,4\% não saíram de casa nos últimos 7 dias. Os idosos que não saíram de casa nos últimos 7 dias eram mais velhos, predominantemente do sexo feminino, menos independentes e apresentavam mais dificuldades no desempenho de ABVD ou AIVD, precisando e recebendo ajuda de alguém de fora de casa, do que aqueles que saíram para realizar atividades essenciais ou para realizar atividades não essenciais. Além disso, o grupo que não saiu de casa nos últimos 7 dias tinha menor escolaridade do que os que haviam saído para atividades essenciais. Por fim, esse mesmo grupo apresentava mais dificuldades para ABVD ou AIVD, precisava e recebia ajuda de pessoas de dentro de casa, do que o grupo que saiu para atividades não essenciais (Tabela 1).

Os resultados da análise de regressão multinomial são apresentados na Tabela 2. Idosos que apresentam dificuldades para ABVD ou AIVD, necessitam e recebiam ajuda de pessoa de dentro $(\mathrm{OR}=$ 2,34; IC95\%: 1,25-4,39) ou de fora de casa (OR = 3,94; IC95\%: 2,24-6,92) permaneceram mais em casa nos últimos 7 dias. Isso não foi visto entre aqueles que apresentam dificuldades para ABVD ou AIVD, precisam, mas não recebiam ajuda. O aumento da idade também aumentou a chance de não sair de casa nos últimos 7 dias (OR = 1,06; IC95\%: 1,03-1,09) e ser do sexo masculino diminuiu esta chance $(\mathrm{OR}=0,48$; IC95\%: 0,33-0,70). As saídas para atividades essenciais foram maiores na Região Sul do Brasil (OR = 1,77; IC95\%: 1,01-3,10).

Na Figura 1, são apresentadas as probabilidades preditas da permanência em casa nos últimos 7 dias de acordo com a dificuldade, necessidade e recebimento de ajuda para ABVD ou AIVD, por idade. A probabilidade de permanecer em casa nos últimos 7 dias aumentou com a idade em todos os grupos. Entretanto, aqueles que possuem dificuldades, precisam e recebem ajuda de pessoas de fora ou de dentro de casa, bem como os que têm dificuldades, precisam, mas não recebem ajuda estavam mais distanciados socialmente do que os idosos independentes ou os que têm dificuldades, mas não precisam de ajuda para desempenhá-las. 
Tabela 1

Características sociodemográficas de acordo com o estado de distanciamento social em 4.035 participantes do inquérito telefônico do Estudo Longitudinal da Saúde dos Idosos Brasileiros (iniciativa ELSI-COVID-19), 26 de maio a 08 de junho de 2020.

\begin{tabular}{|c|c|c|c|c|}
\hline & $\begin{array}{c}\text { Total } \\
(\mathrm{N}=4.035)\end{array}$ & $\begin{array}{l}\text { Saiu para atividades } \\
\text { não essenciais } \\
(n=580) 14,4 \%\end{array}$ & $\begin{array}{l}\text { Saiu para atividades } \\
\text { essenciais } \\
\text { (n= 1.502) } 37,2 \%\end{array}$ & $\begin{array}{c}\text { Não saiu de casa nos } \\
\text { últimos } 7 \text { dias } \\
(n=1.953) 48,4 \%\end{array}$ \\
\hline Idade [DP] & $70,3 \pm 7,6$ & $68,5 \pm 6,7$ & $67,9 \pm 5,9$ & 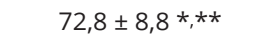 \\
\hline \multicolumn{5}{|l|}{ Sexo [\% (IC95\%)] } \\
\hline Masculino & $44,5(42,5-48,5)$ & $53,0(44,5-61,3)$ & $51,1(46,2-55,8)$ & $37,3(33,5-41,3) *, \star *$ \\
\hline \multicolumn{5}{|l|}{ Escolaridade (anos) [\% (IC95\%)] } \\
\hline Mais de 8 & $36,7(31,3-42,5)$ & $39,0(28,6-50,7)$ & $41,9(35,7-48,3)$ & $30,8(24,6-37,8)$ \\
\hline $5-8$ & $27,2(23,7-31,1)$ & $29,7(22,9-37,4)$ & $29,8(25,4-34,6)$ & $23,9(19,8-28,5)$ \\
\hline $1-4$ & $25,7(23,3-28,2)$ & $24,0(19,1-29,6)$ & $22,1(19,3-25,2)$ & $29,8(26,6-33,1) * *$ \\
\hline Analfabeto & $10,4(8,0-13,3)$ & $7,3(3,7-13,8)$ & $6,2(4,3-8,8)$ & $15,6(12,6-19,1) * *$ \\
\hline \multicolumn{5}{|l|}{ Grande região [\% (IC95\%)] } \\
\hline Norte & $4,4(1,7-11,4)$ & $4,1(1,3-12,1)$ & $4,7(1,7-12,2)$ & $4,4(1,5-11,7)$ \\
\hline Nordeste & $25,2(16,1-37,1)$ & $23,3(12,7-38,7)$ & $23,6(14,9-35,2)$ & $27,5(17,4-40,4)$ \\
\hline Sudeste & $42,7(30,2-56,1)$ & $48,2(33,3-63,5)$ & $42,5(29,7-56,4)$ & $40,8(28,1-54,9)$ \\
\hline Sul & $17,3(8,5-31,8)$ & $12,7(6,8-22,6)$ & $19,5(9,9-35,0)$ & $16,6(7,1-34,1)$ \\
\hline Centro-oeste & $10,4(5,0-20,6)$ & $11,7(5,1-24,2)$ & $9,7(4,1-21,5)$ & $10,7(5,6-19,5)$ \\
\hline \multicolumn{5}{|l|}{ ABVD/AIVD } \\
\hline Independentes & $77,9(73,1-82,0)$ & $88,0(82,4-92,0)$ & $83,5(77,2-88,3)$ & $68,7(62,1-74,6) *, \star * *$ \\
\hline $\begin{array}{l}\text { Possuíam dificuldade e não precisavam de } \\
\text { ajuda }\end{array}$ & $5,1(3,8-6,8)$ & $3,4(1,5-7,5)$ & $6,2(4,0-9,6)$ & $4,7(3,6-6,1)$ \\
\hline $\begin{array}{l}\text { Possuíam dificuldade, precisavam e recebiam } \\
\text { ajuda de pessoa de dentro de casa }\end{array}$ & $5,4(3,7-7,7)$ & $2,7(1,6-4,5)$ & $3,4(2,0-5,9)$ & $8,2(5,7-11,7)$ * \\
\hline $\begin{array}{l}\text { Possuíam dificuldade, precisavam e recebiam } \\
\text { ajuda de pessoa de fora de casa }\end{array}$ & $8,8(6,9-11,1)$ & $3,1(2,2-4,5)$ & $5,3(2,9-9,4)$ & $14,2(11,2-17,8) *, * *$ \\
\hline $\begin{array}{l}\text { Possuíam dificuldade, precisavam e não } \\
\text { recebiam ajuda }\end{array}$ & $2,8(1,4-5,7)$ & $2,7(1,3-5,9)$ & $1,4(0,5-4,1)$ & $4,2(1,7-9,7)$ \\
\hline
\end{tabular}

ABVD: atividades básicas da vida diária; AIVD: atividades instrumentais da vida diária DP: desvio padrão; IC95\%: intervalo de 95\% de confiança. Notas: os dados são apresentados em forma de média e DP, bem como proporções e IC95\%. Todas as estimativas consideraram os parâmetros amostrais e os pesos para aqueles indivíduos que participaram do inquérito telefônico. Atividades não essenciais incluíram: sair para fazer exercícios, encontrar os amigos e outras razões. Atividades essenciais incluíram: sair para comprar remédios, atendimento de saúde e pagar contas. Significância estatística foi aceita quando $\mathrm{p}<0,05$.

* Estatisticamente diferente do grupo que saiu para atividades não essenciais;

** Estatisticamente diferente do grupo que saiu para atividades essenciais. 
Tabela 2

Modelo final de regressão multinomial para análise da associação entre dificuldade e necessidade de ajuda em atividades básicas ou instrumentais da vida diária e distanciamento social nos últimos sete dias, em 4.035 participantes do inquérito telefônico do Estudo Longitudinal da Saúde dos Idosos Brasileiros (iniciativa ELSI-COVID-19), 26 de maio a 8 de junho de 2020.

\begin{tabular}{|c|c|c|}
\hline & $\begin{array}{l}\text { Saiu para atividades essenciais } \\
\text { OR (IC95\%) }\end{array}$ & $\begin{array}{l}\text { Não saiu de casa nos últimos } 7 \text { dias } \\
\text { OR (IC95\%) }\end{array}$ \\
\hline Idade (anos) & $0,98(0,95-1,01)$ & $1,06(1,03-1,09)$ * \\
\hline \multicolumn{3}{|l|}{ Sexo } \\
\hline Feminino & 1,00 & 1,00 \\
\hline Masculino & $0,94(0,63-1,40)$ & $0,48(0,33-0,70)$ * \\
\hline \multicolumn{3}{|l|}{ Escolaridade (anos) } \\
\hline Mais de 8 & 1,00 & 1,00 \\
\hline $5-8$ & $0,88(0,53-1,49)$ & $1,01(0,60-1,70)$ \\
\hline $1-4$ & $0,83(0,51-1,34)$ & $1,27(0,80-2,02)$ \\
\hline Analfabeto & $0,76(0,42-1,38)$ & $1,63(0,80-3,33)$ \\
\hline \multicolumn{3}{|l|}{ Regiões } \\
\hline Sudeste & 1,00 & 1,00 \\
\hline Sul & $1,77(1,01-3,10)$ * & $1,44(0,73-2,84)$ \\
\hline Centro-oeste & $0,96(0,55-1,68)$ & $1,06(0,58-1,93)$ \\
\hline Norte & $1,25(0,48-3,26)$ & $1,27(0,56-2,88)$ \\
\hline Nordeste & $1,16(0,71-1,88)$ & $1,18(0,65-2,14)$ \\
\hline \multicolumn{3}{|l|}{ ABVD/AIVD } \\
\hline Independentes & 1,00 & 1,00 \\
\hline Possuíam dificuldade e não precisavam de ajuda & $2,11(0,86-5,19)$ & $1,25(0,55-2,82)$ \\
\hline $\begin{array}{l}\text { Possuíam dificuldade, precisavam e recebiam ajuda de } \\
\text { pessoa de dentro de casa }\end{array}$ & $1,43(0,77-2,69)$ & $2,34(1,25-4,39)$ * \\
\hline $\begin{array}{l}\text { Possuíam dificuldade, precisavam e recebiam ajuda de } \\
\text { pessoa de fora de casa }\end{array}$ & $1,99(0,88-4,48)$ & $3,94(2,24-6,92)$ * \\
\hline Possuíam dificuldade, precisavam e não recebiam ajuda & $0,55(0,13-2,32)$ & $1,24(0,35-4,35)$ \\
\hline
\end{tabular}

ABVD: atividades básicas da vida diária; AIVD: atividades instrumentais da vida diária; IC95\%: intervalo de 95\% de confiança; OR: odds ratio. Nota: atividades essenciais incluíram: sair para comprar remédios, atendimento de saúde e pagar contas.

* $\mathrm{p}<0,05$ 


\section{Figura 1}

Probabilidade predita da permanência em casa nos últimos 7 dias de acordo com a dificuldade, necessidade e recebimento de ajuda para ABVD ou AIVD, por idade, em idosos da comunidade participantes do inquérito telefônico do Estudo Longitudinal da Saúde dos Idosos Brasileiros (iniciativa ELSI-COVID-19).
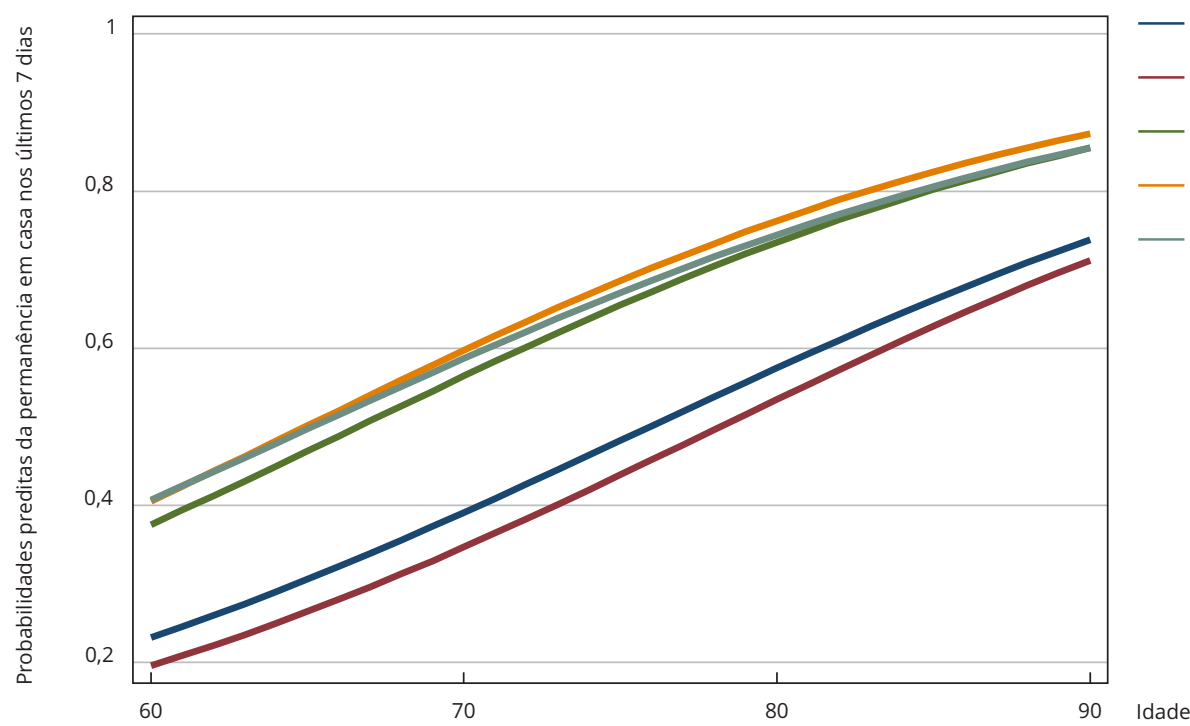

ABVD: atividades básicas da vida diária; AIVD: atividades instrumentais da vida diária.

\section{Discussão}

Nossos principais resultados mostraram que idosos que apresentavam dificuldade para ABVD ou AIVD, necessitavam e recebiam ajuda de pessoas de dentro ou de fora de casa permaneceram mais distanciados socialmente nos últimos 7 dias. Por outro lado, isso não aconteceu para aqueles idosos que apresentaram dificuldade para ABVD ou AIVD, precisavam, mas não recebiam ajuda para realizar essas atividades.

A perda da capacidade funcional traz implicações físicas, psicológicas e sociais para os idosos 19,20, 21,22. Mesmo antes da pandemia, os cuidados com essa população estavam em um cenário frágil e com desigualdades de cuidado 23.

Dessa forma, por se tratar de um grupo de risco, algumas estratégias têm sido adotadas. Idosos que precisam de intervenções clínicas, mas não necessariamente do recebimento de ajuda para desempenhar as ABVD ou AIVD, têm sido acompanhados por meio de teleconsultas 24. Entretanto, nem tudo foi equacionado, pois os idosos que necessitam e recebem ajuda podem estar mais expostos à contaminação pelo novo Coronavírus trazido por terceiros que lhes prestam ajuda 11, e os idosos que também apresentam dificuldades, mas não recebem ajuda, podem estar ainda mais marginalizados quanto ao acesso a cuidados e apoios essenciais. Todo esse cenário é preocupante, pois trata-se de uma população com mais comorbidades, mais propensa a complicações e hospitalizações prolongadas, sobrecarregando ainda mais os serviços de saúde neste momento de pandemia 19,20,23.

De acordo com os resultados do presente estudo, os homens estavam menos distanciados socialmente quando comparados com as mulheres, e à medida em que aumentou a idade, o estado de distanciamento social aumentou. Esses dados corroboram o estudo de Alsan et al. 23 envolvendo 5.198 indivíduos nos Estados Unidos. Os autores apontaram que as maiores diferenças no conhecimento e comportamento relacionado à COVID-19 foram associadas ao sexo e a idade, tendo os homens e os indivíduos com menos de 55 anos uma maior propensão para sair de casa. Já as saídas dos idosos 
para realizar atividades essenciais foram informadas com maior frequência entre os residentes na Região Sul, onde as medidas de distanciamento estavam mais flexíveis, uma vez que a incidência da COVID-19 era menor no momento das entrevistas do que em outras regiões do país 25,26,27,28.

O distanciamento social vem sendo utilizado como estratégia para a prevenção à pandemia 9 . Entretanto, para a sua implementação devem ser consideradas as condições particulares de vulnerabilidade dos segmentos da sociedade, como condição socioeconômica, saúde física e mental, ambiente e percepção de conforto das residências 9,26 .

Estudos com simulações numéricas 11,27 testaram a eficácia das intervenções de distanciamento social em grupos estratificados por idade e apontaram que o distanciamento social seletivo se mostrou ineficaz para a contenção e o controle de novos casos de COVID-19. Por outro lado, o distanciamento social ampliado, se aplicado com a mesma intensidade em todas as faixas etárias, se mostrou significativamente eficaz no achatamento da curva de crescimento da doença.

Dessa maneira, os resultados apresentados neste estudo sugerem que o distanciamento social seletivo, uma opção para populações de risco, parece não ser uma estratégia preventiva efetiva para idosos com dependência funcional e necessidade de ajuda para ABVD ou AIVD, uma vez que estes indivíduos podem estar expostos à contaminação de portadores, por ora assintomáticos, que lhes prestam ajuda.

Este trabalho apresenta três pontos fortes. Primeiro, o estudo foi conduzido em uma grande amostra representativa nacional. Segundo, este é o primeiro estudo a analisar dados de inquéritos telefônicos sobre COVID-19 analisando o estado de distanciamento social em função das dificuldades e necessidades de ajuda para ABVD e AIVD no Brasil. Terceiro e último, todas as informações sobre a COVID-19 foram realizadas sem expor os participantes aos riscos de contaminação, inerentes às entrevistas presenciais.

Por outro lado, reconhecemos como limitação o fato da pesquisa ter sido realizada por meio de entrevistas telefônicas, especificamente para aquelas chamadas que não foram atendidas, recusadas e naquelas em que os números de telefone não eram válidos. Contudo, para minimizar tal limitação, foram usados pesos especificamente derivados para os participantes do inquérito telefônico.

\section{Conclusão}

Idosos que precisam de ajuda de pessoas de dentro ou de fora de casa para desempenhar ABVD ou AIVD não saíram de casa nos últimos 7 dias. Mesmo com o distanciamento social, idosos que recebem ajuda de pessoas não distanciadas não têm a sua exposição à COVID-19 reduzida, o que enfraquece a teoria do distanciamento social seletivo. 


\section{Colaboradores}

D. C. Oliveira, C. M. Oliveira, M. F. Lima-Costa e T. S. Alexandre contribuíram na concepção do desenho do estudo, analisaram os dados e interpretaram os resultados. Todos os autores contribuíram igualmente com a redação e revisão da versão final do manuscrito.

\section{Informações adicionais}

ORCID: Dayane Capra de Oliveira (0000-00015825-4511); Cesar Messias de Oliveira (0000-00024099-4762); Maria Fernanda Lima-Costa (00000002-3474-2980); Tiago da Silva Alexandre (00000003-3791-9793).

\section{Agradecimentos}

Agradecemos a todos os funcionários, participantes e financiadores do ELSI-Brasil.O estudo de base do ELSI-Brasil foi apoiado pelo Ministério da Saúde do Brasil (Departamento de Ciência e Tecnologia da Secretaria de Ciência, Tecnologia e Insumos Estratégicos - DECIT/SCTIE, processo 404965/20121); Coordenação de Saúde do Idoso, Departamento de Ações Estratégicas e Programáticas da Secretaria de Atenção à Saúde (COSAPI/DAPES/SAS processos 20836, 22566 e 23700) e Ministério da Ciência, Tecnologia, Inovação e Comunicação do Brasil. O presente trabalho foi realizado com apoio da Coordenação de Aperfeiçoamento de Pessoal de Nível Superior (CAPES), código de financiamento 001.

\section{Referências}

1. Morley JE, Vellas B. COVID-19 and older adult. J Nutr Health Aging 2020; 24:364-5.

2. Wu F, Zhao S, Yu B, Chen Y-M, Wang W, Song $\mathrm{Z}-\mathrm{G}$, et al. A new coronavirus associated with human respiratory disease in China. Nature 2020; 579:265-9.

3. World Health Organization. Coronavirus disease (COVID-19): situation reports - 172 . https://www.who.int/emergencies/diseases/ novel-coronavirus-2019/situation-reports (acessado em 11/Jul/2020).

4. Leonardi M, Padovani A, McArthur JC. Neurological manifestations associated with COVID-19: a review and a call for action. J Neurol 2020; 267:1573-6.

5. Grupo Força Colaborativa COVID-19 Brasil. Orientações sobre diagnóstico, tratamento e isolamento de pacientes com COVID-19. https://sbpt.org.br/portal/orientacoes-co vid-19/ (acessado em 11/Jul/2020).

6. Singh AK, Singh A, Singh R, Misra A. Hydroxychloroquine in patients with COVID-19: a systematic review and meta-analysis. Diabetes Metab Syndr 2020; 14:589-96.

7. Mesa Vieira C, Franco OH, Gómez Restrepo C, Abela T. COVID-19: the forgotten priorities of the pandemic. Maturitas 2020; 136:38-41.

8. Garcia LP, Duarte E. Intervenções não farmacológicas para o enfrentamento à epidemia da COVID-19 no Brasil. Epidemiol Serv Saúde 2020; 29:e2020222.

9. Qualls N, Levitt A, Kanade N, Wright-Jegede N, Dopson S, Biggerstaff M, et al. Community mitigation guidelines to prevent pandemic influenza - United States, 2017. MMWR Recomm Rep 2017; 66:1-34.

10. Duczmal LH, Almeida ACL, Duczmal DB, Alves CRL, Magalhães FCO, Lima MS, et al. Vertical social distancing policy is ineffective to contain the COVID-19 pandemic. Cad Saúde Pública 2020; 36:e00084420.

11. Aprahamian I, Cesari M. Geriatric syndromes and SARS-COV-2: more than just being old. J Frailty Aging 2020; 9:127-9.

12. Qual a diferença de distanciamento social, isolamento e quarentena? Telessaúde RS-UFRGS. https://www.ufrgs.br/telessauders/posts_co ronavirus/qual-a-diferenca-de-distanciamen to-social-isolamento-e-quarentena/ (acessado em 11/Set/2020).

13. Lawton MP, Brody EM. Assessment of older people: self-maintaining and instrumental activities of daily living. Gerontologist 1969; 9:179-86.

14. Katz S, Ford AB, Moskowitz RW, Jackson BA, Jaffe MW, et al. Studies of illness in the aged: a standardized measure of biological and psychosocial function. JAMA 1963; 185:914-9.

15. Lima-Costa MF, Andrade FB, Souza Jr. PRB, Neri AL, Duarte YAO, Castro-Costa E, et al. The Brazilian Longitudinal Study of Aging (ELSI-Brazil): objectives and design. Am J Epidemiol 2018; 187:1345-53. 
16. Nunes JD, Saes MO, Nunes BP, Siqueira FCV, Soares DC, Fassa MEG, et al. Indicadores de incapacidade funcional e fatores associados em idosos: estudo de base populacional em Bagé, Rio Grande do Sul. Epidemiol Serv Saúde 2017; 26:295-304.

17. Lima-Costa MF, Macinko J, Andrade FB, Souza Jr. PRB, Vasconcellos MTL, Oliveira CM. ELSI-COVID-19 initiative: methodology of the telephone survey on coronavirus in the Brazilian Longitudinal Study of Aging. Cad Saúde Pública 2020; 36 Suppl 3:e00183120.

18. Farías-Antúnez S, Lima NP, Bierhals IO, Gomes AP, Vieira LS, Tomasi E. Incapacidade funcional para atividades básicas e instrumentais da vida diária: um estudo de base populacional com idosos de Pelotas, Rio Grande do Sul, 2014. Epidemiol Serv Saúde 2018; 27:e2017290.

19. Alexandre TS, Corona LP, Nunes DP, Santos JLF, Duarte YAO, Lebrão ML. Gender differences in incidence and determinants of disability in activities of daily living among elderly individuals: SABE study. Arch Gerontol Geriatr 2012; 55:431-7.

20. Alexandre TS, Corona LP, Nunes DP, Santos JLF, Duarte YAO, Lebrão ML. Disability in instrumental activities of daily living among older adults: gender differences. Rev Saúde Pública 2014; 48:379-89.

21. Pan American Health Organization. Policy brief: the impact of COVID-19 on older persons. http://www.paho.org/en/documents/ policy-brief-impact-covid-19-older-persons (acessado em 13/Jul/2020).

22. Conselho Federal de Fisioterapia e Terapia Ocupacional. Resolução no 516, de 20 de março de 2020. Dispõe sobre a suspensão temporária do Artigo 15, inciso II e Artigo 39 da Resolução COFFITO no 424/2013 e Artigo 15, inciso II e Artigo 39 da Resolução COFFITO no 425/2013 e estabelece outras providências durante o enfrentamento da crise provocada pela Pandemia do COVID-19. Diário Oficial da União 2020; 23 mar.
23. Alsan M, Stantcheva S, Yang D, Cutler D. Disparities in coronavirus 2019 reported incidence, knowledge, and behavior among US adults. JAMA Netw Open 2020; 3:e2012403.

24. Rio Grande do Sul. Decreto no 55.240 , de 10 de maio de 2020. Institui o Sistema de Distanciamento Controlado para fins de prevenção e de enfrentamento à epidemia causada pelo novo Coronavírus (COVID-19) no âmbito do Estado do Rio Grande do Sul, reitera a declaração de estado de calamidade pública em todo o território estadual e dá outras providências. Diário Oficial do Estado do Rio Grande do Sul 2020; 10 mai.

25. Agência de Notícias do Paraná. Estado impõe medidas mais restritivas para conter avanço da Covid. http://www.aen.pr.gov.br/modules/ noticias/article.php?storyid=107669 (acessado em 15/Jul/2020).

26. Secretaria de Vigilância em Saúde, Ministério da Saúde. Boletim Epidemiológico Especial 20. Doença pelo coronavírus COVID-19. https:// coronavirus.saude.gov.br/boletins-epidemio logicos (acessado em 15/Jul/2020).

27. Armitage R, Nellums LB. The COVID-19 response must be disability inclusive. Lancet Public Health 2020; 5:e257.

28. Matrajt L, Leung T. Evaluating the effectiveness of social distancing interventions to delay or flatten the epidemic curve of coronavirus disease. Emerging Infect Dis 2020; 26:1740-8. 
Abstract

To analyze whether the older adults with difficulty or need of help to perform basic or instrumentals activities of daily living are more socially distanced in times of COVID-19. A total of 4,035 older adults participated in the telephone interviews from the second wave of the Brazilian Longitudinal Study of Aging (ELSI-Brazil). Difficulty, need and receiving help were classified into: (1) independents; (2) had difficulty without need for care; (3) had difficulty, needed and have received care from someone within their household; (4) had difficulty, needed and have received care from someone outside their household; and (5) had difficulty and needed care but did not receive it. Social distancing was categorized as follows: did not leave their houses in the last 7 days, left their houses for essential activities and went out for non-essential activities. Multinomial regression model adjusted for age, sex, schooling and great geographical region was performed. Older adults who had difficulty, needed and have received help from within their homes (odds ratio- $O R=2.34$ 95\%CI: 1.25-4.39) or from outside their homes $(O R=3.94 ; 95 \% C I: 2.24-6.92)$ were more socially distanced. Age increased the odds of not going out $(O R=1.06$; 95\%CI: 1.03-1.09) while be men reduced it $(\mathrm{OR}=0.48 ; 95 \% \mathrm{CI}: 0.33-0.70)$. Living in the South of Brazil has increased the odds of the respondents going out for essential activities (OR = 1.77; 95\%CI: 1.01-3.10). Older adults who had difficulty, needed and have received help from within or outside their homes did not leave their homes in the last 7 days. Even with social distancing, these older adults can not have their exposure to COVID-19 reduced, weakening the theory of selective social distancing.

Social Isolation; Disability; COVID-19; Caring; Aged

\section{Resumen}

Analizar si los ancianos con dificultad o necesidad de ayuda para desempeñar actividades básicas $(A B V D)$ o instrumentales de la vida diaria (AIVD) están más distanciados socialmente en tiempos de COVID-19. Participaron en las entrevistas telefónicas 4.035 ancianos de la encuesta de la segunda fase del Estudio Brasileño Longitudinal del Envejecimiento (ELSI-Brasil). Se clasificó la dificultad, necesidad y recepción de ayuda en: (1) independientes; (2) con dificultad y no necesita ayuda; (3) con dificultad, necesita y recibe ayuda de una persona de dentro de casa; (4) con dificultad, necesita y recibe ayuda de persona de fuera de casa; $y$ (5) con dificultad, necesita, pero no recibe ayuda. El distanciamiento social fue categorizado como: no salió de casa en los últimos 7 días, salió de casa para actividades esenciales y salió de casa para actividades no esenciales. Se usó un modelo de regresión multinomial controlado por edad, sexo, escolaridad y región de Brasil. Permanecieron más distanciados socialmente los ancianos que presentaban dificultad, necesitaban y recibian ayuda de una persona de dentro (odds ratio $O R=2,34$; IC95\%: 1,25-4,39) o de fuera de casa $(O R=3,94$; IC95\%: 2,24-6,92). La edad aumentó la oportunidad de no salir de casa $(O R=1,06$; IC95\%: 1,03-1,09) y el sexo masculino disminuyó $(\mathrm{OR}=0,48$; IC95\%: 0,33-0,70). Estar en la Región Sur aumentó la oportunidad de que los ancianos salgan para actividades esenciales $(O R=1,77$; IC95\%: 1,01-3,10). Los ancianos que necesitan ayuda de personas de dentro o de fuera de casa no salieron de casa en los últimos 7 días. Incluso con distanciamiento social, los ancianos que reciben ayuda de personas no distanciadas no tienen su exposición reducida a la COVID-19, lo que debilita la teoría del distanciamiento social selectivo.

Aislamiento Social; Incapacidad; COVID-19; Cuidar; Anciano
Recebido em 21/Jul/2020

Versão final reapresentada em 15/Set/2020

Aprovado em 09/Out/2020 